\title{
Trends in 1-year survival of people admitted to hospital in Ontario, 1994-2009
}

\author{
Carl van Walraven MD MSc
}

\section{Abstract \\ Background: Changes in the long-term survival of people admitted to hospital is unknown. This study examined trends in 1-year survival of patients admitted to hospital adjusted for improved survival in the general population.}

Methods: One-year survival after admission to hospital was determined for all adults admitted to hospital in Ontario in 1994, 1999, 2004, or 2009 by linking to vital statistics datasets. Annual survival in the general population was determined from life tables for Ontario.

Results: Between 1994 and 2009, hospital use decreased (from $8.8 \%$ to $6.3 \%$ of the general adult population per year), whereas crude 1year mortality among people with hospital admissions increased (from $9.2 \%$ to $11.6 \%$ ). During this time, patients in hospital became significantly older (median age increased from 51 to $58 \mathrm{yr}$ ) and sicker (the proportion with a Charlson comorbidity index score of 0 decreased

$\mathrm{H}$ ospitals have a special place in most health care systems. Hospital staff care for the people with the most serious illnesses and the most vulnerable. They are frequently the location of many life-defining moments - including birth, surgery, acute medical illness and death - of many people and their families. Hospitals serve as a focus in the training of most physicians. In addition, they consume a considerable proportion of health care expenditures worldwide. ${ }^{1}$

Given the prominence of hospitals in health care systems, measuring outcomes related to hospital care is important. In particular, the measurement of trends for outcomes of hospital care can help us to infer whether the care provided to hospital patients is improving. Previous such studies have focused on survival trends for specific diseases or patients who received treatment in specific departments. ${ }^{2-12}$ None of these studies have adjusted for survival trends in the general population, the adjustment for which is important to determine whether changes in survival of patients in hospital merely reflect changes in the overall from $68.2 \%$ to $60.0 \%$ ), and were more acutely ill on admission (elective admissions decreased from $47.4 \%$ to $42.0 \%$; proportion brought to hospital by ambulance increased from $16.1 \%$ to $24.8 \%$ ). Compared with 1994, the adjusted odds ratio (OR) for death at 1 year in 2009 was 0.78 (95\% confidence interval [CI] 0.77-0.79). However, 1-year risk of death in the general population decreased by $24 \%$ during the same time. After adjusting for improved survival in the general population, risk of death at 1 year for people admitted to hospital remained significantly lower in 2009 than in 1994 (adjusted relative

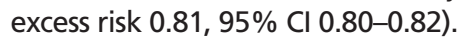

Interpretation: After accounting for both the increased burden of patient sickness and improved survival in the general population, 1-year survival for people admitted to hospital increased significantly from 1994 to 2009. The reasons for this improvement cannot be determined from these data.

population. In this study, whether or not patient outcomes have changed over time was determined by examining trends in 1-year survival in all patients admitted to hospital, adjusting for improved survival in the general population.

\section{Methods}

\section{Study design and data sources}

This study used 2 population-based administrative databases to identify patients admitted to hospital in Ontario during the study period: the Discharge Abstract Database, which records demographic and diagnostic information on all nonpsychiatric admissions to hospital, and the Registered Persons Database, which records the date of death for all residents of Ontario.

\section{Study period and population}

Population life tables for Ontario were used to measure trends in 1-year survival of adults admitted to hospital relative to the general population. At the time of the study, 2009 was the latest year for which life tables were available. To measure
Competing interests: None declared.

This article has been peer reviewed.

Correspondence to: Carl van Walraven, carlv@ohri.ca

CMAJ 2013. DOI:10.1503 /cmaj.130875 
trends in survival of patients admitted to hospital, 5-year decrements from 2009 were selected to the earliest year possible given the data available in the Discharge Abstract Database and Registered Persons Database (i.e., 1991).

The Discharge Abstract Database was used to identify all adults (defined as age $>20 \mathrm{yr}$ ) admitted to any Ontario acute care hospital in 1994, 1999, 2004 or 2009. Exclusions included sameday surgeries, admissions to psychiatric facilities (because their data are captured in another data set), and admissions to in-patient rehabilitation or long-term care facilities (because they are distinct from acute care hospitals). In addition, patients ineligible for health care coverage in Ontario were excluded because the capture of their outcomes would be incomplete. Only the first admission for each person in each year was used in the analysis.

\section{Outcome measures}

The primary outcome was all-cause mortality within 1 year of admission to hospital. This outcome was chosen instead of in-hospital mortality to account for potential temporal changes in several factors, including patient discharge thresholds (which could decrease over time as lengths of stay decrease, thereby potentially decreasing in-hospital mortality at the expense of an increased risk of death after discharge) and home-based palliative care services after admission to hospital (the increased prevalence of such services over time and increasing prevalence of dying at home for patients with recent stays in hospital ${ }^{13}$ could decrease the risk of death in hospital). The Registered Persons Database was used to determine whether patients died within the year following their admission date.

Covariates were identified from each person's Discharge Abstract Database record and included age, sex, urgency of the admission (elective or urgent), whether the patient arrived by ambulance, admission service (medical, surgical or obstetricsgynecology) and comorbidity. Comorbidity was measured using the Charlson comorbidity index score, as calculated by diagnoses coded in each person's index Discharge Abstract Database record, as well as those coded for all admissions to hospital within the previous year. To calculate the final Charlson comorbidity index score, the International Classification of Diseases, 9th revision, clinical modification (ICD-9-CM), and International Classification of Diseases, 10th revision (ICD-10), diagnostic codes were grouped into the Charlson medical conditions using methods from Quan and colleagues ${ }^{14}$ and weights from Schneeweiss and colleagues. ${ }^{15}$ Finally, admissions were categorized into primary diagnosis groups using the ICD-9-CM code for their most responsible diagnosis; to do this for the 2004 and 2009 cohorts, ICD-10 codes were first converted to ICD-9-CM using "cross-walks" from the Canadian Institute for Health Information.

\section{Statistical analysis}

Ontario adult population estimates from Statistics Canada for each study year were used to calculate rates of hospital use. Multivariate binomial logistic regression was used to measure the independent association of admission year with risk of death at 1 year adjusted for patient factors (age, sex and Charlson comorbidity index score) and admission covariates (urgency, service and ambulance status). To further delineate trends in survival, this model was repeated for secondary outcomes, including death in hospital and death after discharge (up to 1 year after admission).

Ontario life tables for each study year were used to determine the annual risk of death in the general population for all age-sex strata. Methods from Dickman and colleagues ${ }^{16,17}$ were used to measure the unadjusted 1-year risk of death for patients in hospital relative to the Ontario population. This unadjusted relative survival was calculated as the ratio of observed survival (calculated as $e^{-x}$, where $x$ is the observed number of deaths divided by the total observation time) to expected survival (determined from provincial life tables). To compare relative survival between years, standardized weighted mean relative survival was calculated using weights based on age proportions from all 4 years in the study period.

Multivariate relative survival models were then used to measure trends of 1-year survival in patients in hospital, adjusting for patient-level covariates, as well as the improved survival of the general population. Relative survival models are additive hazards models in which the total hazard (or risk) of death in the patient group is the sum of the known hazard in the general population and the excess hazard in the patient group. ${ }^{17}$ The hazard for the general population was determined from Ontario life tables. The parameter estimates for the full relative survival model were determined with a Poisson error structure in SAS 9.3 ${ }^{17}$ and were exponentiated to calculate the relative excess risk. ${ }^{18}$ The relative excess risk quantifies the change in death risk relative to the comparator group, adjusted for model covariates and changes in population survival over time.

\section{Results}

During the 4 years studied, a total of 3616026 nonpsychiatric admissions to hospital for adults 
:

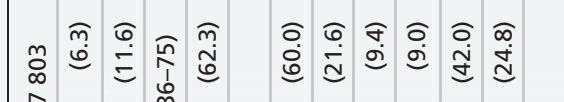

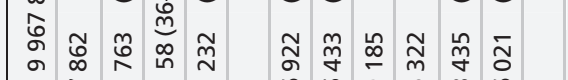

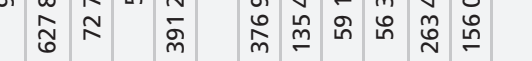

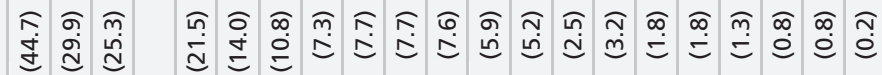
ळī $\stackrel{\infty}{\infty} \stackrel{\infty}{\stackrel{\infty}{\leftrightarrow}}$

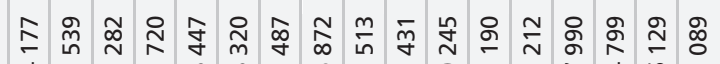

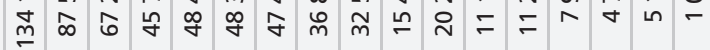

孛

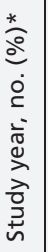

㝵

品

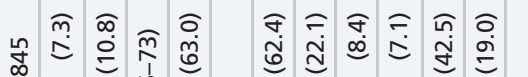

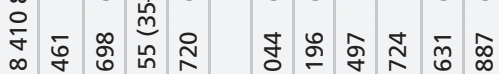

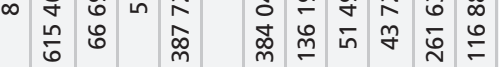

สุ

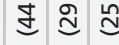

สำ 용

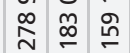

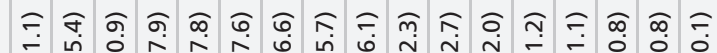

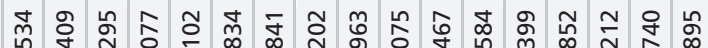
色

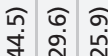
욜

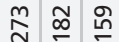

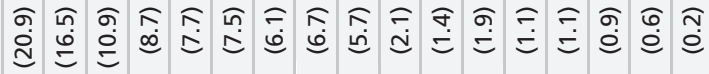

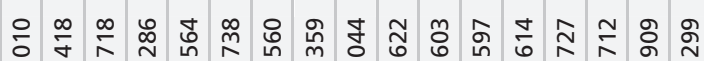

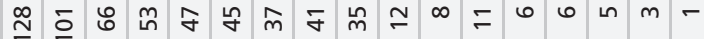

莺

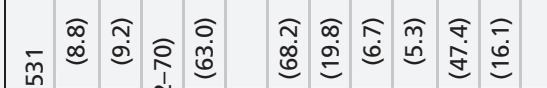

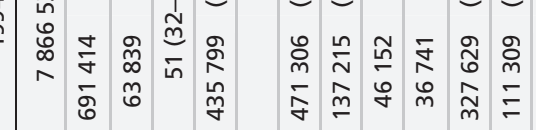

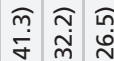

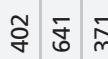
$\stackrel{\mathscr{\infty}}{\infty} \underset{\mathbb{N}}{\mathbb{\infty}}$

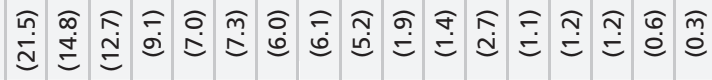
స్

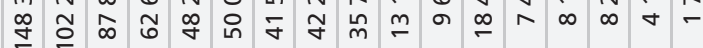


occurred in Ontario. Of these admissions, 1060123 were excluded because they were repeat admissions, for a total of 2555903 adult residents of Ontario with at least 1 admission during the study period. Population-based rates of admission to hospital decreased significantly between 1994 and $2009\left(8.8 \%\right.$ v. $6.3 \%$, Mantel-Haenszel $\chi^{2}$ for trend $41478, p<0.001$ ) (Table 1).

From 1994 to 2009, crude mortality 1 year

Table 2: Odds of death within 1 year of admission to hospital

\begin{tabular}{|c|c|c|c|}
\hline $\begin{array}{l}\text { Factor } \\
\text { Study year }\end{array}$ & $\begin{array}{c}\text { Model A: } \\
\text { Adjusted OR }(95 \% \mathrm{Cl})\end{array}$ & \multicolumn{2}{|c|}{$\begin{array}{c}\text { Model B: } \\
\text { Relative survival, * } \\
\text { excess risk }(95 \% \mathrm{Cl})\end{array}$} \\
\hline 1994 & 1.00 (ref) & 1.00 & (ref) \\
\hline 1999 & $0.92(0.91-0.94)$ & 0.93 & $(0.91-0.94)$ \\
\hline 2004 & $0.90(0.89-0.91)$ & 0.92 & $(0.91-0.93)$ \\
\hline 2009 & $0.78(0.77-0.79)$ & 0.81 & $(0.80-0.82)$ \\
\hline \multicolumn{4}{|l|}{ Patient age, yr } \\
\hline $20-25$ & 1.00 (ref) & 1.00 & (ref) \\
\hline $25-29$ & $1.09(0.98-1.22)$ & 1.16 & $(1.03-1.30)$ \\
\hline $30-34$ & $1.37(1.24-1.51)$ & 1.44 & $(1.30-1.60)$ \\
\hline $35-39$ & $1.95(1.78-2.13)$ & 1.96 & $(1.77-2.16)$ \\
\hline $40-44$ & $2.69(2.46-2.94)$ & 2.53 & $(2.30-2.78)$ \\
\hline $44-49$ & $3.25(2.98-3.54)$ & 2.90 & $(2.65-3.19)$ \\
\hline $50-55$ & $3.94(3.62-4.29)$ & 3.33 & $(3.04-3.65)$ \\
\hline $55-59$ & $4.56(4.19-4.96)$ & 3.69 & $(3.36-4.04)$ \\
\hline $60-64$ & $5.42(4.98-5.89)$ & 4.11 & $(3.76-4.51)$ \\
\hline $65-69$ & 6.37 (5.86-6.92) & 4.50 & $(4.11-4.93)$ \\
\hline $70-75$ & $7.54(6.94-8.19)$ & 4.87 & $(4.44-5.33)$ \\
\hline $75-79$ & 9.28 (8.54-10.1) & 5.31 & $(4.85-5.81)$ \\
\hline $80-84$ & $11.9(11.0-13.0)$ & 5.85 & $(5.34-6.40)$ \\
\hline $85-89$ & $16.0(14.8-17.4)$ & 6.44 & $(5.88-7.06)$ \\
\hline $90-94$ & $25.0(23.0-27.2)$ & 8.08 & $(7.37-8.86)$ \\
\hline$\geq 95$ & $43.8(40.1-47.9)$ & 10.58 & $(9.59-11.7)$ \\
\hline Female sex & $0.8(0.79-0.81)$ & 0.89 & $(0.88-0.91)$ \\
\hline \multicolumn{4}{|c|}{$\begin{array}{l}\text { Charlson comorbidity index } \\
\text { score }\end{array}$} \\
\hline 0 & 1.00 (ref) & 1.00 & (ref) \\
\hline $1-2$ & $2.85(2.81-2.89)$ & 4.93 & $(4.82-5.04)$ \\
\hline $3-4$ & $4.60(4.53-4.67)$ & 8.87 & $(8.66-9.08)$ \\
\hline$\geq 5$ & $17.0(16.8-17.3)$ & 26.8 & $(26.2-27.4)$ \\
\hline Elective admission & $0.57(0.56-0.58)$ & 0.54 & $(0.54-0.55)$ \\
\hline Arrival by ambulance & $1.70(1.68-1.71)$ & 1.58 & $(1.56-1.60)$ \\
\hline \multicolumn{4}{|l|}{ Admitting service } \\
\hline Medicine & 1.00 (ref) & 1.00 & (ref) \\
\hline Surgery & $0.63(0.63-0.64)$ & 0.62 & $(0.61-0.63)$ \\
\hline Obstetrics-Gynecology & $0.22(0.20-0.23)$ & 0.20 & $(0.19-0.22)$ \\
\hline
\end{tabular}

Note: $\mathrm{Cl}=$ confidence interval, $\mathrm{OR}=$ odds ratio.

* Relative excess risk quantifies risk of death relative to the comparator group adjusted for model covariables and changes in population survival over time. after admission increased progressively from $9.2 \%$ to $11.6 \%$ ( $\chi^{2}$ for trend $2247, p<0.001$ ) (Table 1). However, patients admitted to hospital during this time were progressively older and sicker (Table 1): the median age of patients increased from 51 to 58 years, the proportion of patients with scores of 0 on the Charlson comorbidity index decreased from $68.2 \%$ to $60.0 \%$, the proportion of patients admitted electively decreased from $47.4 \%$ to $42.0 \%$, and the proportion of patients brought to hospital by ambulance increased from $16.1 \%$ to $24.8 \%$. Overall, the proportion of patients within each primary diagnosis group was consistent throughout the study.

After adjusting for changes in patient characteristics over the years studied, the 1-year risk of death decreased significantly between 1994 and 2004. Independent of patient age, sex and comorbidity, admission urgency and service, and ambulance status, the 1-year adjusted odds of death were $22 \%$ lower in 2009 than in 1994 (adjusted odds ratio [OR] 0.78, 95\% CI 0.77-0.79) (Table 2, model A). The adjusted relative odds of death increased notably with patient age (adjusted OR $43.8,95 \%$ CI $40.1-47.9$ in patients aged $\geq 95 \mathrm{yr}$ v. patients aged $<25 \mathrm{yr}$ ) and comorbidity burden (adjusted OR 17.0, 95\% CI 16.8-17.3 for patients with Charlson comorbidity index score $\geq 5$ v. 0 ). Compared with patients admitted to medical wards, the adjusted odds of death were $37 \%$ lower in patients admitted to surgical wards (adjusted OR $0.63,95 \%$ CI $0.63-0.64$ ) and $78 \%$ lower in patients admitted to an obstetrics-gynecology service (adjusted OR $0.22,95 \%$ CI $0.20-0.23$ ). The logistic model had excellent discrimination ( $C$ statistic 0.896 ) and very good calibration (Appendix 1, available at www.cmaj.ca/lookup/suppl/doi :10.1503/cmaj.130875/-/DC1). Significant improvements between 1994 and 2009 were also seen when the model outcome was either death in hospital or death after discharge (Appendix 2, available at www.cmaj.ca/lookup/suppl/doi:10 .1503/cmaj.130875/-/DC1).

\section{Changes in survival in the general population}

The annual risk of death in the general population notably decreased during the study period for most age groups (Figure 1A). During all 4 years studied, the annual risk increased significantly with patient age, but decreased throughout the study period (relative to 1994 values) within almost all age strata (Figure 1B). For people under 50 years of age, the annual risk of death (relative to 1994) decreased by $18 \%$ in 1999 , $22 \%$ in 2004 and 28\% in 2009 (data not shown). More consistent separation in survival between the years was seen in people 50 years of age and 
older: relative to 1994, the average relative death risk in this age group decreased progressively by $4 \%$ in 1999, 14\% in 2004 and 22\% in 2009 (data not shown).

\section{Survival of patients in hospital relative to the general population}

Survival of patients discharged from hospital relative to the general population decreased as patients aged. For women, the average relative survival (over all 4 years studied) was $99.8 \%$ for women 20 years of age, $94.8 \%$ for women 50 years of age and $80.3 \%$ in women 80 years of age, respectively (data not shown). Relative survival was significantly lower for men, with corresponding values of 98.8\%, $93.4 \%$ and $73.6 \%$ (data not shown). Unadjusted relative survival in the entire population (accounting for the significantly increased age of the population in hospital in 2009 v. 1994) decreased slightly for both women $(92.1 \%$ in $1994 \mathrm{v}$. $91.2 \%$ in 2009) and men (89.2\% in 1994 v. $88.5 \%$ in 2009) (data not shown).

Relative survival of patients in hospital was poorest in strata defined by covariables that increased the risk of death. For example, unadjusted relative survival decreased as Charlson

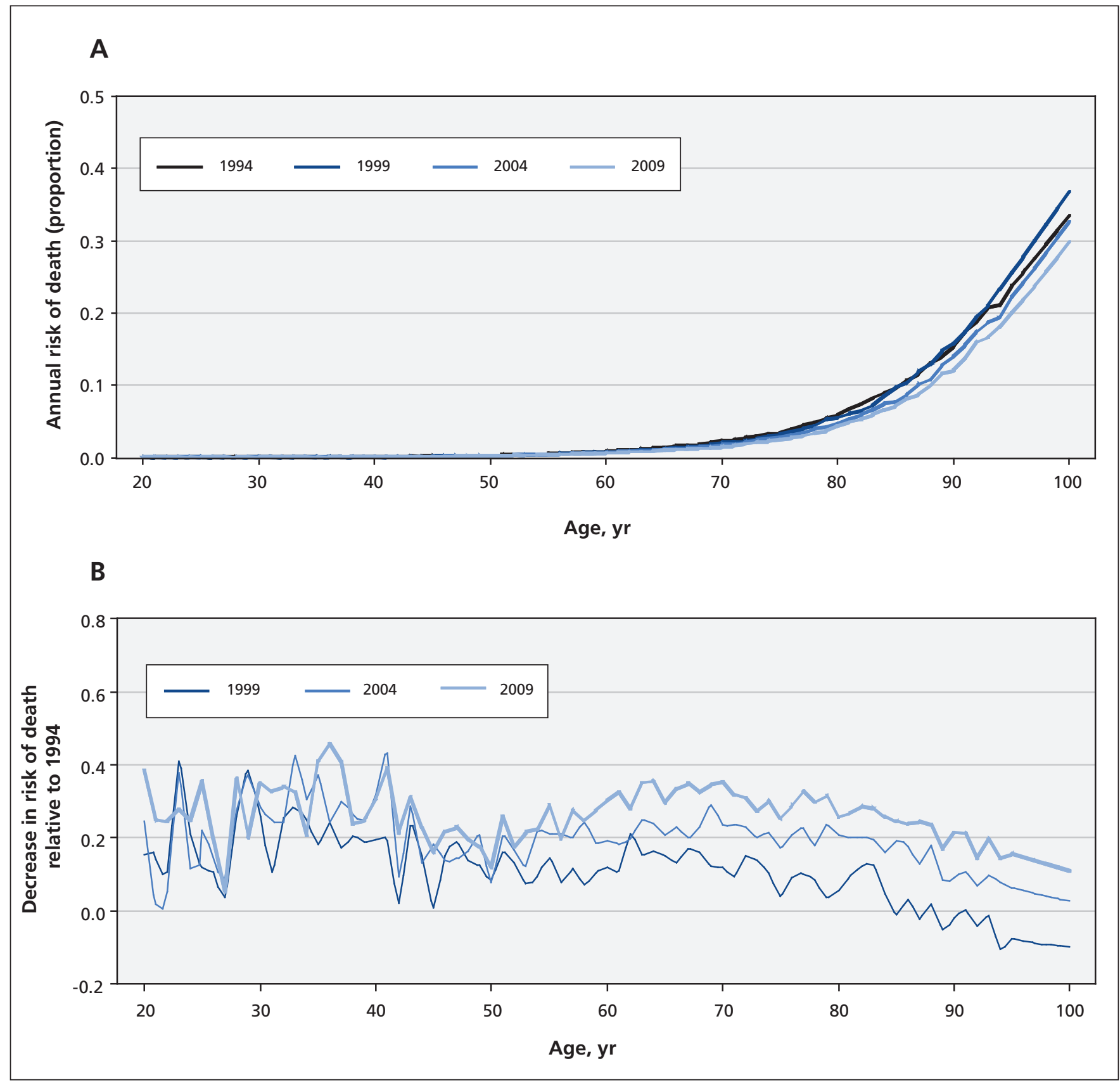

Figure 1: Annual risk of death for adults in Ontario, 1994-2009. (A) Annual risk of death in each study cohort year for all adults. (B) Decrease in annual risk of death relative to 1994. 
comorbidity index score increased (Table 3). Similar patterns were seen for admission urgency, ambulance status and admission service (Table 3). After standardizing for significant changes in the age structure of patients admitted to hospital, unadjusted relative survival changed minimally within all covariable strata.

Significant increases in 1-year survival for patients in hospital were seen in the adjusted relative survival model (Table 2, model B). After accounting for notable improvements in survival for the general population (Figure 1B) and adjusting for the important covariables that influence patient outcomes (Table 1), 1-year risk of death for patients admitted to hospital decreased significantly throughout the study period (Table 4). Improved adjusted relative survival was consistent in all patient strata but appeared to vary significantly by patient age and Charlson comorbidity index score. Improved relative survival between 1994 and 2009 was particularly prominent in younger patients, as patient comorbidity increased, and in patients with diseases of the circulatory system, nervous system and blood or blood-forming organs, as well as infectious or parasitic diseases.

\section{Interpretation}

Hospital care is common and consumes a large proportion of health care resources. It is therefore important to measure trends in outcomes of

\begin{tabular}{|c|c|c|c|c|}
\hline \multirow[b]{2}{*}{ Patient group } & \multicolumn{4}{|c|}{ Study year, relative survival, $\%$} \\
\hline & 1994 & 1999 & 2004 & 2009 \\
\hline \multicolumn{5}{|l|}{$\begin{array}{l}\text { Charlson comorbidity } \\
\text { index score }\end{array}$} \\
\hline 0 & 93.9 & 93.4 & 93.4 & 93.4 \\
\hline $1-2$ & 87.1 & 86.0 & 86.2 & 86.1 \\
\hline $3-4$ & 83.7 & 82.2 & 82.3 & 82.2 \\
\hline$\geq 5$ & 84.8 & 83.4 & 83.6 & 83.5 \\
\hline \multicolumn{5}{|l|}{ Admission urgency } \\
\hline Elective & 94.4 & 94.0 & 94.0 & 94.0 \\
\hline Urgent & 88.4 & 87.4 & 87.5 & 87.3 \\
\hline \multicolumn{5}{|l|}{ Arrival by ambulance } \\
\hline No & 92.6 & 92.0 & 92.1 & 92.1 \\
\hline Yes & 84.5 & 83.0 & 83.1 & 82.9 \\
\hline \multicolumn{5}{|l|}{ Admitting service } \\
\hline Medicine & 87.0 & 85.9 & 86.0 & 85.9 \\
\hline Surgical & 90.5 & 89.7 & 89.8 & 89.8 \\
\hline Obstetrics-Gynecology & 98.3 & 98.2 & 98.1 & 98.1 \\
\hline
\end{tabular}

patients admitted to hospital to help gauge the performance of this component of the health care system. This retrospective cohort study found that, between 1994 and 2009, hospital use in Ontario decreased, but patient sickness increased. In addition, the crude 1-year risk of death for patients with hospital admissions increased, but decreased when adjusted for important confounders. During the same period, all-cause risk of death in the general population decreased, but this trend did not explain the improved 1-year adjusted survival seen in patients with hospital admissions. This study identifies important improvements in health outcomes in a very common patient population.

A study by Ford and colleagues ${ }^{19}$ attributed reduced mortality trends in coronary artery disease to medical treatment and risk factor modification. These mechanisms could also explain improved survival in patients admitted to hospital. Improved survival may reflect advances in medical care since the early 1990s, such as interventions for acute coronary syndromes, ${ }^{20}$ aggressive use of statin and antiplatelet therapy in chronic coronary artery disease, ${ }^{20}$ the use of angiotensin-converting enzyme (ACE) inhibitors, angiotensin-receptor blockers and $\beta$ blockers for congestive heart failure, ${ }^{20}$ implantable cardioverter defibrillators for sudden cardiac death, ${ }^{20}$ aromatase inhibitors and other hormone treatments for breast cancer, ${ }^{21}$ and effective antiretroviral therapy for AIDS. ${ }^{22}$ This trend may also reflect increased uptake of evidence-based treatments. For example, studies have shown increased use of evidence-based treatments for cardiovascular diseases including $\beta$-blockers, statins, ACE inhibitors and angiotensin-receptor blockers in patients after myocardial infarction ${ }^{23}$ and in patients with congestive heart failure. ${ }^{24}$

In addition to advances in treatments and increased use of such improvements, the positive effects of preventive health strategies might further explain these results. It is possible that a greater focus on lifestyle modifications over the study period contributed to the improved outcomes of patients admitted to hospital.

\section{Limitations}

The earliest date of the study was 1994 owing to data availability. It is possible that improved patient survival would have been more extensive if one had been able to look back further in time. In addition, it is uncertain whether these results are generalizable to other jurisdictions. Because the data required for this study are available in many countries, these analyses could be replicated in other provinces and countries to deter- 
mine whether similar results are seen elsewhere.

It cannot be determined whether - and to what extent - the increased patient Charlson comorbidity score is due to more complete diagnostic coding over time. If this potential bias was pervasive, then the odds of death would be

Table 4: Changes in adjusted excess risk of death at 1 year relative to 1994 in specific patient groups

Excess risk of death, adjusted* $\mathrm{HR}(95 \% \mathrm{Cl})$

Patient group

Overall

Age, yr

$<50$
$50-69$
$70-79$

70-79

$\geq 80$

Sex

Female

Male

Charlson comorbidity index score

(0

1-2

3-4

$\geq 5$

Admission urgency

Elective

Urgent

Arrival by ambulance

No

Yes

Admitting service

Medicine

Surgery

Obstetrics-Gynecology

Primary diagnosis group

Diseases of the circulatory system

Diseases of the digestive system

Diseases of the genitourinary system

Neoplasms

Injury and poisoning

Diseases of musculoskeletal system

Diseases of the respiratory system

Symptoms, signs and ill-defined conditions

Endocrine, nutritional, metabolic and immune diseases

Factors influencing health status and contact with health services

Infectious and parasitic diseases

Mental disorders

Diseases of the skin and subcutaneous tissue

Diseases of the blood and blood-forming organs
Diseases of the nervous system and sense organs

1999

$0.926 \quad(0.914-0.939)$

2004

2009

$0.846 \quad(0.807-0.888)$

$0.870 \quad(0.849-0.891)$

$0.930 \quad(0.909-0.952)$

1.002 (0.978-1.026)

$0.918(0.906-0.930)$

$0.806(0.796-0.897)$

$0.868(0.828-0.911)$

$0.875(0.854-0.896)$

$0.920(0.899-0.942)$

0.977 (0.955-1.000)

0.780 (0.743-0.819)

$0.759(0.741-0.777)$

$0.797(0.778-0.816)$

$0.883(0.864-0.903)$

$0.931 \quad(0.913-0.949)$

$0.941(0.924-0.959)$

$0.825(0.809-0.840)$

0.924 (0.907-0.941)

0.897 (0.881-0.914)

$0.789(0.775-0.803)$

$\begin{array}{ll}1.088 & (1.032-1.148) \\ 0.919 & (0.897-0.942) \\ 0.936 & (0.911-0.962) \\ 0.890 & (0.873-0.908)\end{array}$

$1.203(1.144-1.264)$

$0.992(0.968-1.017)$

1.201 (1.143-1.262)

$0.861(0.840-0.883)$

$0.886(0.862-0.911)$

$0.786(0.765-0.808)$

0.890

$0.937 \quad(0.924-0.950)$

$0.846(0.830-0.863)$

$0.750(0.736-0.765)$

$0.957 \quad(0.920-0.995)$

$0.939(0.926-0.952)$

$0.839(0.828-0.851)$

$0.891(0.855-0.929)$

$0.656(0.628-0.684)$

$0.899 \quad(0.883-0.915)$

$0.918(0.901-0.935)$

$0.802(0.787-0.817)$

0.961 (0.943-0.980)

0.926 (0.909-0.944)

$0.830(0.815-0.845)$

$0.961 \quad(0.943-0.980)$

$0.926(0.909-0.944)$

$0.830(0.815-0.845)$

$0.946 \quad(0.914-0.979)$

$0.884(0.854-0.915)$

$0.709(0.684-0.734)$

$0.903 \quad(0.751-1.086)$

1.182 (0.992-1.409)

$0.829(0.689-0.997)$

\begin{tabular}{llllll}
0.903 & $(0.880-0.926)$ & 0.865 & $(0.843-0.888)$ & $0.713(0.694-0.733)$ \\
\hline 0.980 & $(0.930-1.033)$ & $0.979(0.930-1.030)$ & $0.840(0.799-0.883)$ \\
\hline 1.006 & $(0.923-1.097)$ & $1.019(0.940-1.106)$ & $0.874(0.808-0.945)$ \\
\hline 0.913 & $(0.892-0.935)$ & $0.903(0.882-0.925)$ & $0.778(0.759-0.797)$ \\
\hline 1.123 & $(1.053-1.198)$ & $1.085(1.017-1.158)$ & $0.987(0.927-1.051)$ \\
\hline 1.103 & $(0.954-1.276)$ & $1.014(0.875-1.176)$ & $0.770(0.664-0.893)$ \\
\hline 0.984 & $(0.947-1.022)$ & $1.099(1.059-1.141)$ & $1.006(0.970-1.044)$ \\
\hline 1.071 & $(0.999-1.149)$ & $1.131(1.057-1.209)$ & $1.063(0.995-1.136)$ \\
\hline 0.960 & $(0.888-1.039)$ & $0.976(0.905-1.052)$ & $0.864(0.802-0.931)$ \\
\hline 1.026 & $(0.955-1.103)$ & $1.030(0.964-1.101)$ & $1.379(1.296-1.467)$ \\
\hline 1.038 & $(0.928-1.161)$ & $0.813(0.728-0.907)$ & $0.701(0.628-0.783)$ \\
\hline 0.743 & $(0.681-0.810)$ & $0.894(0.825-0.969)$ & $0.727(0.675-0.783)$ \\
\hline 1.074 & $(0.956-1.207)$ & $1.073(0.957-1.204)$ & $1.051(0.944-1.169)$ \\
\hline 1.369 & $(1.145-1.637)$ & $1.532(1.288-1.822)$ & $1.328(1.118-1.579)$ \\
\hline 1.072 & $(0.966-1.191)$ & $0.934(0.842-1.035)$ & $0.734(0.661-0.816)$
\end{tabular}

Note: $\mathrm{Cl}=$ confidence interval, $\mathrm{HR}=$ hazard ratio.

*Adjusted for all other covariables listed in Table 2, as well as improved survival in the general population over time (Figure 1B). 
biased down for later years in the adjusted model. This problem is unlikely to explain the study's findings for 2 reasons: almost all hospital physicians who were clinically active during the study period would state that patients have become sicker over time; and the stratified model suggested that patients with a Charlson comorbidity index score of 0 had a significantly worse outcome over time (Table 4), a result that would not be expected with "up-coding," because patients in each of the index's strata would be getting healthier over time.

Finally, it is possible that changes in admission patterns could explain some decrease in risk of death over time. For example, patients with a poor prognosis (such as an undefined metastatic cancer or a palliative case) may have been more likely to be admitted to hospital (for work-up or palliation) in 1994 than in 2009. This would increase the prevalence of patients with a poor prognosis in early years of study observation. However, given that survival improvements were seen in a broad assortment of patient populations (Table 4), it is unlikely that such potential changes would explain the study's results.

\section{Conclusion}

This study noted significant improvement between 1994 and 2009 in survival of patients admitted to hospital in Ontario. Because admissions to hospital are so common, and the drop in the risk of death is so extensive, these changes translate into a meaningful decrease in life-years lost.

\section{References}

1. Anderson GF. In search of value: an international comparison of cost, access and outcomes. Health Aff (Millwood) 1997;16:163-71.

2. Joynt KE, Orav EJ, Jha AK. Mortality rates for Medicare beneficiaries admitted to critical access and non-critical access hospitals, 2002-2010. JAMA 2013;309:1379-87.

3. Kaboli PJ, Go JT, Hockenberry J, et al. Associations between reduced hospital length of stay and 30-day readmission rate and mortality: 14-year experience in 129 Veterans Affairs hospitals. Ann Intern Med 2012;157:837-45.

4. Lenihan CR, Montez-Rath ME, Mora Mangano CT, et al. Trends in acute kidney injury, associated use of dialysis, and mortality after cardiac surgery, 1999 to 2008. Ann Thorac Surg 2013; 95:20-8.

5. Yeung DF, Boom NK, Guo H, et al. Trends in the incidence and outcomes of heart failure in Ontario, Canada: 1997 to 2007. CMAJ 2012;184:E765-73.

6. Coles AH, Fisher KA, Darling C, et al. Recent trends in postdischarge mortality among patients with an initial acute myocardial infarction. Am J Cardiol 2012;110:1073-7.

7. Girotra S, Nallamothu BK, Spertus JA, et al. Trends in survival after in-hospital cardiac arrest. N Engl J Med 2012;367:1912-20.

8. Kirksey M, Chiu YL, Ma Y, et al. Trends in in-hospital major morbidity and mortality after total joint arthroplasty: United States 1998-2008. Anesth Analg 2012; 115:321-7.

9. Lindenauer PK, Lagu T, Shieh MS, et al. Association of diagnostic coding with trends in hospitalizations and mortality of patients with pneumonia, 2003-2009. JAMA 2012;307:1405-13.

10. Anjum A, von Allmen R, Greenhalgh R, et al. Explaining the decrease in mortality from abdominal aortic aneurysm rupture. Br J Surg 2012;99:637-45.

11. Chen J, Normand SL, Wang Y, et al. National and regional trends in heart failure hospitalization and mortality rates for Medicare beneficiaries, 1998-2008. JAMA 2011;306:1669-78.

12. Moxey PW, Hofman D, Hinchliffe RJ, et al. Trends and outcomes after surgical lower limb revascularization in England. $\mathrm{Br}$ J Surg 2011;98:1373-82

13. Wilson DM, Truman CD, Thomas R, et al. The rapidly changing location of death in Canada. Soc Sci Med 2009;68:1752-8.

14. Quan H, Sundararajan V, Halfon P, et al. Coding algorithms for defining comorbidities in ICD-9-CM and ICD-10 administrative data. Med Care 2005;43:1130-9.

15. Schneeweiss S, Wang PS, Avorn J, et al. Improved comorbidity adjustment for predicting mortality in Medicare populations. Health Serv Res 2003;38:1103-20.

16. Dickman PW. Estimating and modelling relative survival using SAS. 2004. Available: http://biostat3.net/download/sas/relative _survival_using_sas.pdf (accessed 2013 Sep. 9).

17. Dickman PW, Sloggett A, Hills M, et al. Regression models for relative survival. Stat Med 2004;23:51-64.

18. Suissa S. Relative excess risk: an alternative measure of comparative risk. Am J Epidemiol 1999;150:279-82.

19. Ford ES, Ajani UA, Croft JB, et al. Explaining the decrease in US deaths from coronary disease, 1980-2000. N Engl J Med 2007; 356:2388-98.

20. Teo KK. Recent advances. Cardiology. BMJ 1998;316:911-5.

21. Howell A, Dowsett M. Recent advances in endocrine therapy of breast cancer. BMJ 1997;315:863-6.

22. Cohn JA. Recent advances. HIV infection - I. BMJ 1997;314: 487-91.

23. Austin PC, Tu JV, Ko DT, et al. Use of evidence-based therapies after discharge among elderly patients with acute myocardial infarction. CMAJ 2008;179:895-900.

24. Lee DS, Mamdani MM, Austin PC, et al. Trends in heart failure outcomes and pharmacotherapy: 1992 to 2000. Am J Med 2004; 116:581-9.

Affiliations: Departments of Medicine and Epidemiology and Community Medicine, University of Ottawa, and the Ottawa Hospital Research Institute, Ottawa, Ont.

Disclaimer: This study was supported by the Institute for Clinical Evaluative Sciences (ICES), which is funded by an annual grant from the Ontario Ministry of Health and LongTerm Care (MOHLTC). The opinions, results and conclusions reported in this paper are those of the authors and are independent from the funding sources. No endorsement by ICES or the Ontario MOHLTC is intended or should be inferred. 